\title{
Defining Residual Radial Translation of Distal Radius Fractures: A Potential Cause of Distal Radioulnar Joint Instability
}

\author{
Mark Ross, MBBS, FRACS, FAOrthA ${ }^{1,2,3}$ Livio Di Mascio, MBBS, FRCS Ed (Tr Ortho) $)^{1,4}$ \\ Susan Peters, BOccThy (Hons) ${ }^{1,3,5}$ Allen Cockfield, MbChb, FRACS ${ }^{1,2}$ \\ Fraser Taylor, BSc, MbChb, FRACS ${ }^{1,2}$ Greg Couzens, MBBS, FRACS, FAOrthA ${ }^{1,2}$
}

\footnotetext{
${ }^{1}$ Brisbane Hand and Upper Limb Research Institute, Brisbane, Australia

2 Orthopaedic Department, Princess Alexandra Hospital, Brisbane, Australia

${ }^{3}$ School of Medicine, The University of Queensland, Brisbane, Australia

${ }^{4}$ Orthopaedic Department, St Bartholomew's and the Royal London

Hospital, London, United Kingdom

${ }^{5}$ School of Health and Rehabilitation Sciences, The University of

Queensland, Brisbane, Australia
}

Address for correspondence Mark Ross, MBBS, FRACS, FAOrthA, Brisbane Hand and Upper Limb Research Institute, 9/259 Wickham Terrace, Brisbane, Queensland, Australia 4000 (e-mail: research@upperlimb.com).

J Wrist Surg 2014;3:22-29.

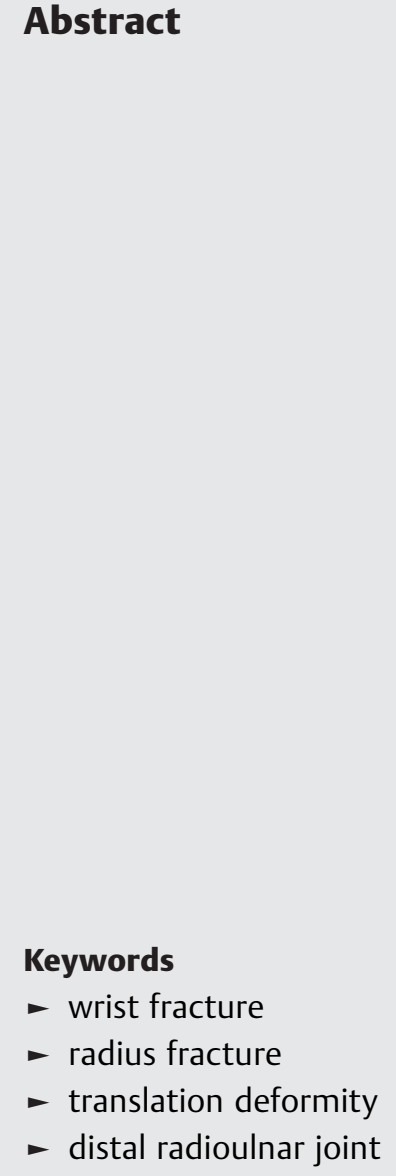

Background Instability of the distal radioulnar joint (DRUJ) is a complication that can occur following distal radius fracture or malunion. We have observed that residual radial translation of the distal radius, relative to the radial shaft, may be a causal factor of DRU] instability, even once the traditional radiographic parameters (volar tilt, radial inclination, and ulnar variance) have been restored. Residual radial translation of the distal fragment may cause detensioning of the distal interosseous membrane (IOM) and pronator quadratus with poor apposition between the ulnar head and sigmoid notch. This may potentially lead to persistent instability of the ulnar head following internal fixation. Residual radial translation deformity is at risk of being overlooked by the wrist surgeon as there is no existing radiographic parameter that accurately measures this deformity.

Patients and Methods In this study, 100 normal wrist radiographs were reviewed by three fellowship-trained orthopedic surgeons to develop a simple and reproducible technique to measure radial translation.

Results Utilizing the method described, the point of intersection between the ulnar cortex of the shaft of the radius and the lunate left a mean average of $45.48 \%$ (range 25 $73.68 \%$ ) of the lunate remaining on the radial side. In the majority of cases more of the lunate resided ulnar to this line. High levels of agreement with inter-rater (intraclass coefficients $=0.967$ ) and intra-rater (intraclass coefficients $=0.79$ ) reliability was observed.

Conclusions The results of this study can be used to define a normal standard against which residual radial translation can be measured to assess the reduction of distal radius fractures. This new parameter aids in the development of surgical techniques to correct residual radial translation deformity. In addition, awareness and correction of this potential malreduction at the time of surgery may decrease the need for other 
procedures on the ulnar side of the wrist to improve DRUJ stability, such as ulnar styloid fixation, TFCC repair, or ligamentous grafting.

Level of Evidence Level II (Diagnostic)

Distal radial fractures are one of the most common fractures of the upper extremity. ${ }^{1,2}$ Instability of the distal radioulnar joint (DRUJ) can occur following such fractures. ${ }^{3}$ Stability of the DRUJ is influenced by the osseous anatomy, DRUJ capsule, palmar and dorsal radioulnar ligaments, triangular fibrocartilage complex, ulnocarpal ligaments, interosseous membrane (IOM), carpi ulnaris, and pronator quadratus., ${ }^{4,5}$ The biomechanics of this joint are complex, and the relative importance of each structure in the maintenance of stability remains controversial. ${ }^{4}$ With advances in the treatment of these fractures that promote stable anatomic fixation (including fragment-specific fixation of the distal radius to gain anatomical reduction and early movement), we have observed a decreased need for ulnar-sided stabilization procedures at the time of distal radial fracture fixation. ${ }^{6}$ When we first began pursuing internal fixation with anatomic reduction of these fractures around 1999, we utilized fragment-specific fixation, which includes a radial buttress implant that is anatomically contoured; when applied to the radial aspect of the distal radius, it automatically corrects radial translation of the distal fragments. Simultaneously, we observed a decrease in instability of the DRUJ intraoperatively, with a decrease in the number of procedures to fix the ulnar styloid or repair the triangular fibrocartilage complex (TFCC). We believed that this observation related to the improved anatomic reduction of the distal radius, but we did not define the specific parameters in fracture reduction that may be related. Later, during our initial experience with fixation of distal radius fractures using volar locked plating systems, we observed an increase in intraoperative instability of the DRUJ that required intraoperative or subsequent procedures on the ulnar side of the wrist. ${ }^{6}$ We examined these cases in detail and postulated that residual radial translation may be the cause. ${ }^{6}$ We believe that the almost obligate correction of this coronal-plane radial translation deformity with the radial buttress plate in the Trimed system (Trimed Inc., Santa Clarita, CA, USA) is the explanation for this marked observed change in the DRUJ stability post reduction. As a corollary, although the anatomic contouring of angle-stable volar plates facilitates reduction of sagittal plane deformity, it does not facilitate correction of coronal plane deformity unless the surgeon is aware of this potential malreduction.

The treatment of DRUJ instability is complex, and several procedures have been described in an attempt to manage this difficult problem. ${ }^{7-9}$ Persistent DRUJ instability following distal radius fracture is often attributed to a disruption of the foveal insertion of the TFCC, which may occur as a purely soft tissue injury but is often identified by the presence of a basiulnar styloid fragment ${ }^{8,9}$ However, DRUJ stability may not be restored even when the ulnar styloid fragment is reduced and the foveal insertion of the TFCC is reattached (-Fig. 1). We have observed cases where Type 3 ulnar styloid fractures have been internally fixed with complete reattachment of the foveal attachment, yet DRUJ instability has persisted in association with residual radial translation (-Fig. 2). Conversely, particularly when restoration of anatomy is achieved, the DRUJ may remain stable even when a large ulnar styloid fragment is not fixed (-Fig. 3 ).

Radiographic parameters previously described, including radial inclination, ulnar variance, and volar tilt, do not accurately describe or measure the malreduction in terms of radial translation. It is hypothesized that radial translation deformity detensions the IOM and pronator quadratus, which may lead to DRUJ instability ${ }^{6}$. To define this potentially problematic malreduction better, we studied the radiographic parameters of normal distal radial radiographs in an attempt to provide a simple and reproducible technique that can be used to identify and evaluate the presence of residual radial translation during or after distal radius fracture treatment. In more recent work, Wolfe and colleagues ${ }^{10}$ have performed cadaveric testing of DRUJ stability and demonstrated that when there was a distinct dorsal oblique band of the IOM, there was a significant decrease in stability if there was radial translation of the distal fragment in simulated distal radius fracture fixation.

We describe this parameter as "radial translation" since all the other parameters for defining reduction of distal radius fractures also reference the relationship between the distal fragments and the radial shaft. Although some of the biomechanical implications of this malreduction relate to ulnar displacement of the radial shaft relative to the ulnar shaft, we believe the description of radial translation of the distal radius is more consistent with existing conventions in describing parameters of distal radial fracture reduction.

In addition, using the radiographic technique we describe, it is easier to measure the malreduction consistently and reproducibly and reference it to radiographs of the uninjured side when there is uncertainty regarding reduction. We have observed that intraoperative radiographs (using fluoroscopy) to assess reduction will show a decrease in the gap between radial and ulnar shafts if there is residual radial translation present, but it is difficult to quantify. It is rare to see widening of the DRUJ intraoperatively following internal fixation. However, trying to measure the space between the radial and ulnar shafts is unpredictable and difficult to standardize with respect to patient size and image planes.

The purpose of this article is to establish a reproducible technique for measuring radial translation that can be readily and easily used to identify the presence of this deformity. 


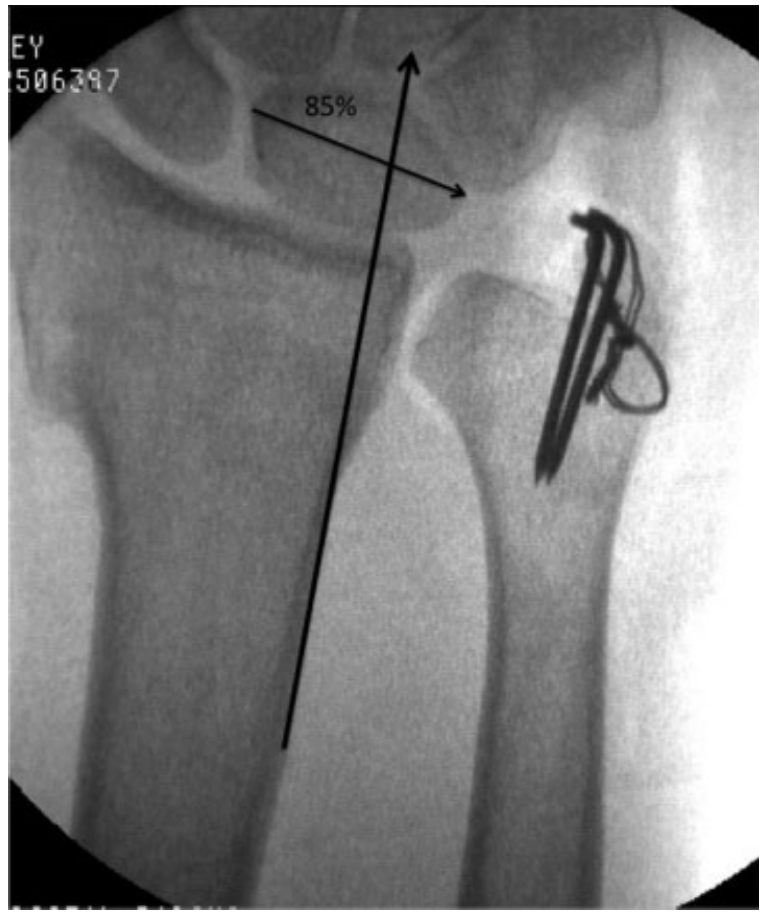

Fig. 1 Radial translation malunion with persistent DRU] instability in spite of ORIF ulnar styloid and transosseous foveal repair of TFCC.

\section{Patients and Methods}

Posteroanterior (PA) radiographs, using a standardized technique, of skeletally mature individuals with anatomically unaltered DRUJs, no history of DRUJ instability, and no associated history of wrist fracture or dislocation were identified from a large radiographic database. Ethics approval was obtained from the appropriate Human Research Ethics Committee Board. The radiographs were examined using a standard Webpacs viewer (Inteleviewer Version 3.7). Radiographs were excluded if any of the following was true:
1. The distal $10 \mathrm{~cm}$ of the radius was not visible.

2. There was evidence of radiocarpal pathology.

3. There was more than 5 degrees of radial or ulnar deviation of the wrist, assessed by deviation of the long axis of the middle metacarpal from that of the radius.

Radial translation was measured by drawing a line along the ulnar aspect of the radial shaft proximal to the metaphyseal flare and extended distally through the proximal row of the carpus on the PA radiograph. This line intersects the lunate. The point of intersection was evaluated by drawing a second line along the transverse width of the lunate on the AP radiograph, parallel to the distal radial articulation. The point of intersection of these two lines was measured from the radial side of the lunate to determine the percentage of lunate radial to this point ( - Fig. 4 ).

A single observer repeated these measurements for all 100 radiographs studied at two separate sittings 1 month apart to evaluate for intraobserver agreement. Interobserver agreement was calculated on 25 radiographs by three fellowshiptrained orthopedic surgeons with an interest in wrist surgery. Each of these surgeons reviewed 25 radiographs to calculate the inter-rater reliability of this parameter. Single-measure intraobserver and interobserver reliability were assessed using the intraclass correlation coefficient (ICC) calculations using a two-way random effects model for absolute agreement (i.e., systematic differences between raters are considered relevant). We used 95\% confidence intervals (CI). ICC values were interpreted as: $>0.75$ was excellent or high level of agreement, $0.40-0.75$ was fair to good level of agreement, and $<0.40$ was considered poor. ${ }^{11}$ Statistical analysis, including ICC calculations, computed with SPSS Version 21.

\section{Results}

One hundred radiographs fulfilling the study inclusion and exclusion criteria were identified. There were 42 females and 58 males, with a mean age of 43 years (range 18-66).

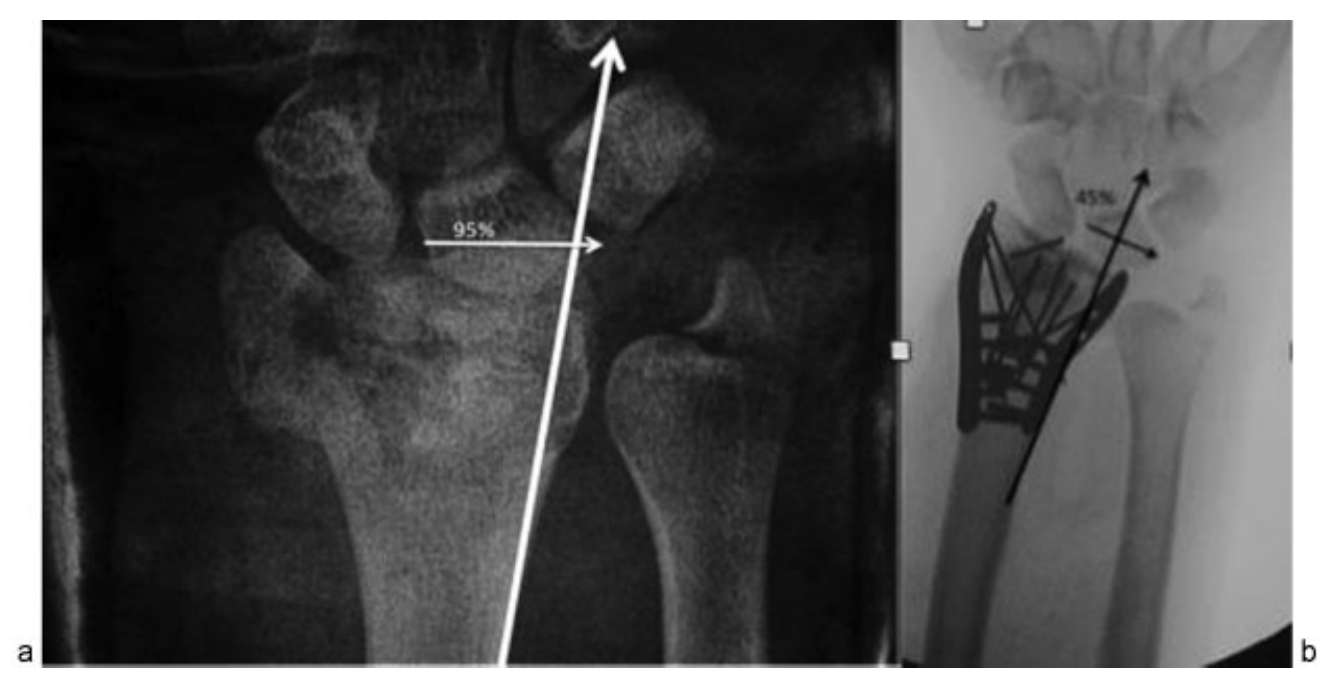

Fig. 2 (a) Radial translation deformity with type 3 ulnar styloid and noted DRUJ instability. No abnormal finding according to Fujitani's classification on pre-operation X-ray. (b) Correction of radial translation deformity and no procedure required on ulnar side. Instability was corrected intraoperatively, and no instability was noted postoperatively. 


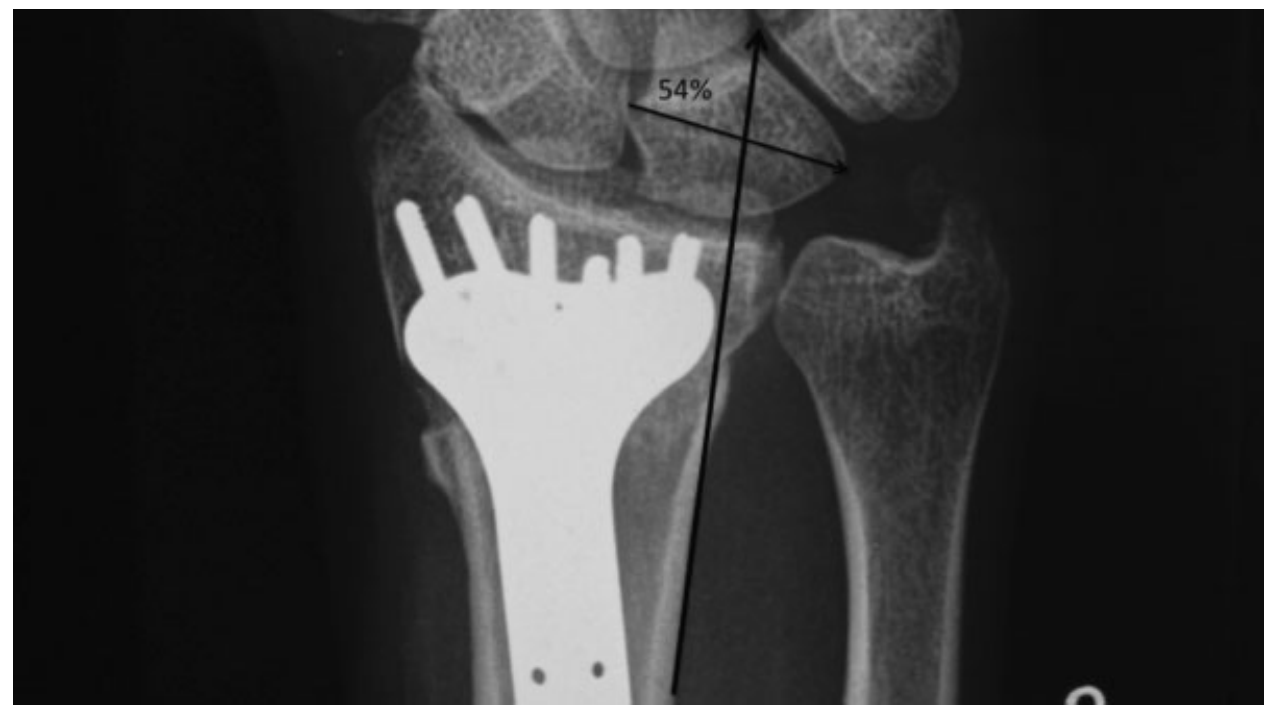

Fig. 3 Radial translation osteotomy for DRUJ instability with successful stability achieved with no ulnar-sided procedure.

For all individuals studied, the point of intersection had a mean of $45.48 \%$ (SD = 9.6\%; range $25-73.68 \%$ ) of the lunate remaining on the radial side and a mode of $50 \%$ (- Fig. 5 ).

Three percent of wrists measured had a shaft intersection point $<30$ percent. Seventy-five percent of wrists measured had a shaft intersection point between $30-50$ percent of the

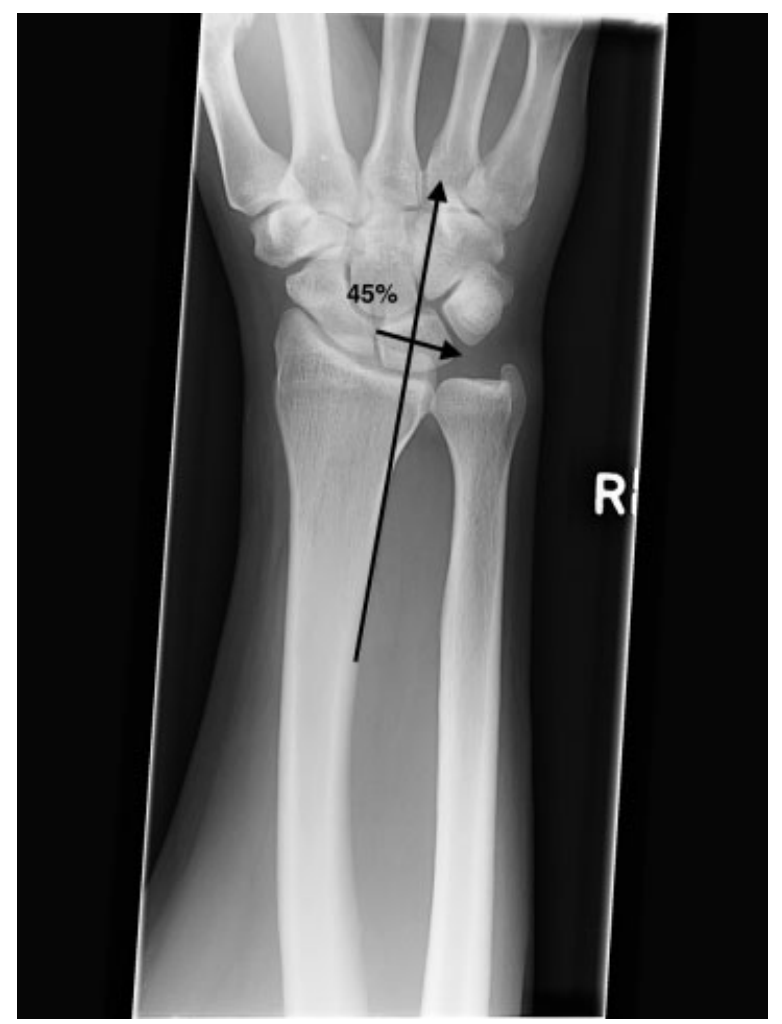

Fig. 4 A line is drawn parallel to the ulnar aspect of the radius proximal to the metaphyseal flare and extrapolated distally into the proximal row of the carpus. The point of intersection within the lunate is evaluated by drawing a second line along the transverse width of the lunate on the AP radiograph that is parallel with the distal radial articulation. The percentage of lunate radial to this line is calculated. lunate, meaning that between $50-70$ percent of the lunate was ulnar to the reference line from the radial shaft. Fourteen percent measured a shaft intersection between 51-60 percent of the lunate. Eight percent of wrists measured had a shaft intersection point $>60$ percent. No shaft intersection points were measured below 25 percent or above 73.68 percent of the lunate width.

\section{Intraobserver Reliability}

The intraclass correlation (ICC) is used to assess the consistency, or conformity, of measurements made by multiple observers measuring the same quantity. Interobserver variability refers to systematic differences among the observer. Intraobserver variability refers to deviations of a particular observer's score. The intraobserver reliability calculation of the shaft intersection point revealed a high level of agreement using ICC and 95\% confidence intervals (95\% CI) using an absolute agreement definition $(p<0.001)$.

ICC $=0.967$, (95\% CI, 0.945 to 0.979$)$

Cronbach's $\alpha$ for the two items is 0.985 , suggesting that the items have relatively high internal consistency. This measures how closely related a set of items are as a group. A "high" value of $\alpha$ is used as a measure that the items reflect the underlying construct that they are attempting to measure.

\section{Interobserver Reliability}

The interobserver (between observer) reliability calculation also revealed a high level of agreement using ICC and 95\% CIs and an absolute agreement definition $(p<0.001)$.

Interobserver reliability was calculated using ICCs as follows:

ICC (Rater 1 and 2): 0.78 (95\% CI, 0.621 to 0.889 )

ICC (Rater 1 and 3): 0.79 (95\%CI, 0.518 to 0.909 )

ICC (Rater 2 and 3): 0.80 (95\% CI, 0.539 to 0.914 )

The Cronbach's $\alpha$ coefficient for the three raters was 0.905 . 


\section{Shaft Intersection \%}

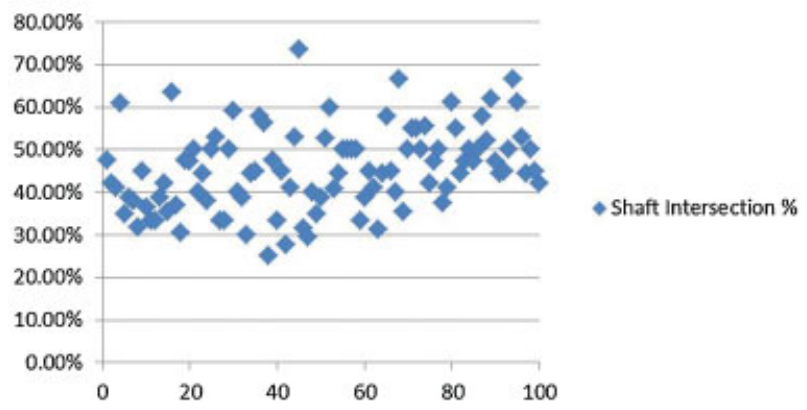

Fig. 5 Percentage of lunate radial to point of intersection of lunate with extrapolated line on ulnar aspect of radial shaft.

\section{Discussion}

With the increasing popularity of volar locked plating systems (used for the treatment of distal radius fractures), the potential for the creation of a stable construct with a radial translation malreduction is ever-present. Angle-stable volar plates are very effective at facilitating correction of radial length and volar tilt. However, they do not automatically correct radial translation, because they are applied to an essentially flat surface without any inherent guide to their positioning in the coronal plane. Radial column buttress plates such as the radial pin plate from Trimed ${ }^{\mathrm{TM}}$ (Trimed Inc, Santa Clarita, CA, USA) do force correction of this deformity. However, the existing classic parameters of distal radial anatomy (radial shortening, palmar inclination, and radial tilt) ${ }^{12}$ do not describe the presence of a postreduction radial translation deformity of the distal fragments. This type of deformity has been noted in the literature previously, albeit variably between pre- and postreduction radiographs. $^{6,12-14}$ The relationship between the distal attachment of the brachioradialis and the "radial beak" pattern of distal radius fractures was seen in $51 \%$ of patients in Koh's series. ${ }^{13}$ However, the relationship is difficult to assess reliably on intraoperative or postoperative radiographs due to comminution of the radial aspect of the fracture line, variability of the radial flare, and obscurity caused by the overlying fixation plate. Due to the deforming force of the brachioradialis on the distal fracture fragment, release of its tendinous insertion has been advocated to aid open reduction, as closed reduction is usually not possible (-Fig. 6). Other techniques for correcting this translational deformity have been described. ${ }^{6,14}$

There is a paucity of literature investigating the importance of this type of deformity and the presence of DRUJ instability.

The technique previously described by Fujitani and coauthors ${ }^{12}$ discussed radial translation as a prognostic indicator for DRUJ instability based on prereduction injury radiographs and made no reference whatsoever to reduction parameters of the fracture. The radial translation was expressed as a ratio of increased DRUJ clear-space to radial shaft dimension proximal to the fracture line. ${ }^{12}$ This technique is complicated and difficult to reproduce. The technique relies on the accurate identification of the cortical margin of the ulna aspect of the sigmoid notch of the radius at the DRUJ and does not comment on whether the dorsal or volar margin should be used in a rotated distal radial fragment. No intra- or interobserver reliability testing was calculated. Furthermore, these landmarks as a ratio to radial width are difficult to calculate, particularly intraoperatively using image intensification. Variations in proximal extent of the fracture line influence the width of the radial shaft relative to the ratio in regard to different fracture patterns. The intraoperative utility is questionable, since the DRUJ clear space (numerator) is very small compared with the radial width (denominator), potentially lowering its sensitivity in an intraoperative environment.

Additionally, in Fujitani's technique ${ }^{12}$ the actual gap radiographically in the DRUJ on films taken at the time of injury may also be influenced by a large number of external factors, particularly with regard to arm positioning. We have observed this phenomenon in our observation of distal radial fracture films and found extreme variation of the DRUJ gap with the same patient, on different images and techniques. In particular, if one attempts to standardize these pictures by taking X-ray images in a 90/90 position, the weight of the arm on the radius will close the gap between the sigmoid notch and the distal ulna, thereby artificially eliminating an abnormal finding.

The fact that the DRUJ interval may be normal in spite of significant persistent radial translation of the distal fragment is very adequately demonstrated by the radiographs in - Fig. 6. In this radiograph, the DRUJ interval is normal even though $87 \%$ of the lunate is lying radial to our defined axis line; when the fracture is reduced and the radial translation is corrected, the radioulnar joint interval remains the same. Fujitani's parameter promotes awareness of the possibility of DRUJ instability based on prereduction radiographs ${ }^{12}$ but offers no specific guidance to the assessment of radial fracture reduction.

Bronstein et al $^{15}$ touched on consideration of a variety of aspects of distal radius fracture reduction, including coronal plane translation. They did not, however, present any method for radiographic measurement of parameters of distal radial fracture reduction. They measured forearm rotation range with differing configurations of distal radial malreduction in a cadaveric model. In regard to translation of the distal radius in the radial direction, they stated that there was no significant difference in rotation with translation of the distal radius up to $10 \mathrm{~mm}$ in the radial direction.

Following distal radius fracture, attention is often turned to the presence of an associated ulnar styloid fracture if persistent DRUJ instability exists after fixation. However, in our experience the radial translation of the distal fragment, with ulnar translation of the proximal radial shaft relative to the ulna, may lead to detensioning of pronator quadratus and the distal oblique bundle (DOB) of the IOM where present. $^{10,16}$ It can be difficult to assess this radial translation deformity in these fractures, due to the lack of bony 


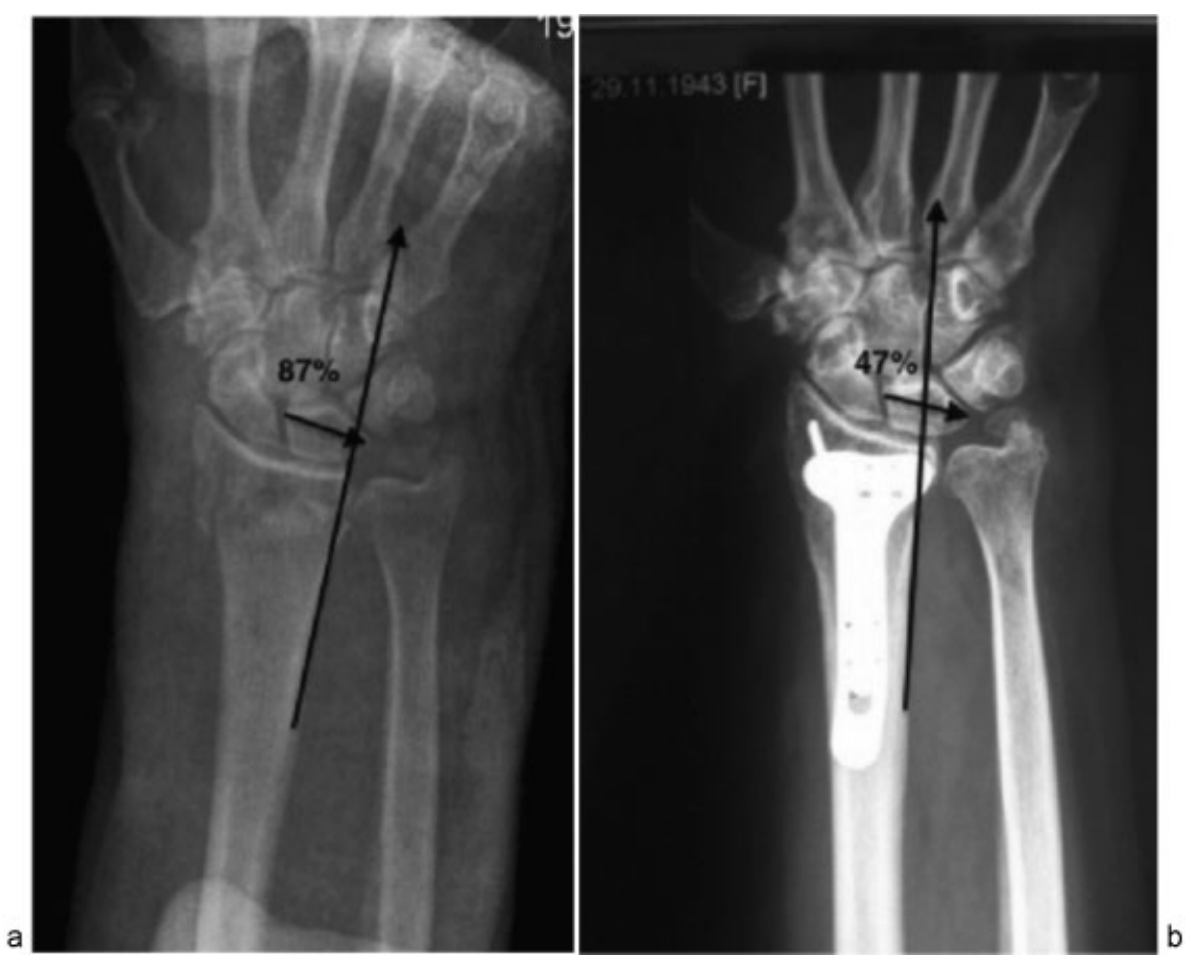

Fig. 6 (a) PA radiographs of a distal radius fracture displaying radial translation deformity. (b) The percentage of the lunate ulnar to the reference limit has been corrected after anatomical open reduction and internal fixation.

landmarks and anatomical variation in the sigmoid notch on plain radiographs. ${ }^{17}$

Awareness of this potential malreduction in distal radial fracture fixation, combined with a simple radiographic tool to assess this parameter, allows radial translation to be assessed and addressed. We recommend that surgeons pay careful attention to this aspect of reduction when internally fixing distal radius fractures. Volar plate fixation has allowed excellent control of all the classically described parameters for assessment of distal radial fracture reduction, particularly in the sagittal plane, yet it has no inherent control over radial translation in the coronal plane. When DRUJ instability is noted intraoperatively after fixation, consideration should be given to correcting any residual radial translation first rather than immediately performing bony or soft tissue repair in the region of the distal ulna or styloid. We have found in our experience that with correction of the bony anatomy anatomically in light of the assessment of the presence of radial translation deformity, any instability of the DRUJ often resolves. This can usually be achieved by fixing the plate initially to the distal fragments and then to the shaft with a single proximal shaft screw and assessing DRUJ instability. Translation can be adjusted using the proximal shaft screw as a fulcrum, and once DRUJ stability is confirmed by translating the distal fragment in an ulnar direction, the remaining shaft screws can be inserted.

In addition, following radius fracture union (after either operative or nonoperative treatment), if DRUJ instability is present, this parameter should be assessed and corrected by radial osteotomy rather than ulnar-sided stabilization surgery. If concerns regarding DRUJ stability exist during fixation of acute fractures, this parameter can also be used to make a quantifiable comparison of reduction with radiographs of the uninjured side. Similar comparisons with the other side can be made when planning corrective osteotomy for DRUJ instability associated with this deformity.

In our clinical experience, we have virtually eliminated the need for ulnar-sided repair in acute fractures by attending to this aspect of reduction. Furthermore, when DRUJ instability is associated with this malunion in healed fractures, we have found corrective osteotomy to be simpler and more predictable than complex ulnar-sided soft tissue reconstructions. We have successfully treated DRUJ instability after radius fracture union with a corrective osteotomy targeting only radial translation of the radius, even when all other classic parameters of reduction are normal. Stability has been achieved without the need for fixation of an associated ulnar styloid fracture or TFCC repair.

Limitations of this study include the fact that although the radiographs in the database were PA radiographs taken according to a consistent standardized protocol for our department, they were not standardized according to the specific published criteria by Metz and colleagues. ${ }^{18}$

Although intraobserver agreement was very high, there was only $78-80 \%$ agreement between observers. Although this is considered to be an excellent outcome in the context of such assessments, ${ }^{11}$ it cannot be ignored that a level of disagreement did exist. However, the clinical purpose of 
this parameter is to determine whether radial translation exists and requires intraoperative correction, and observers were able to determine that radial translation did (or did not) exist in $100 \%$ of cases. The ICC statistics were performed on the exact measurement calculations of the intersection, and a level of disagreement (using an absolute agreement method) would be expected. This study attempts to establish the normal value for the radiographic parameter, and although there is a small level of disagreement between raters, the degree of percentage difference that resulted in classification of a statistical disagreement between observers is small $(<6 \%)$ relative to the range of results. We do not believe that this influences its clinical utility, particularly when the parameter is used to make a comparison with radiographs of the uninjured side. That is, in the patient with a distal radius fracture, does a significant radial translation deformity exist and does it impact on DRUJ instability?

Current research conducted by the authors aims to define the clinical relevance of this radiological parameter in adults with distal radius fractures.

\section{Conclusions}

The results of this study can be used to define a normal standard against which residual radial translation can be measured in the unstable DRUJ associated with distal radius fractures. We have termed this parameter "radial translation." This new parameter aids in the development of surgical techniques to correct residual radial translation deformity. In addition, awareness and correction of this potential malreduction at the time of surgery may decrease the need for other procedures on the ulnar side of the wrist to improve DRUJ stability, such as ulnar styloid fixation, TFCC repair, or ligamentous grafting.

Although preoperative radial translation deformity may be more likely to occur when there is greater disruption of the ulnar-sided stabilizing structures, this makes it even more important for the surgeon to be aware of this deformity and to ensure that from a technical perspective, the radial deformity is corrected at the time of surgery. It may well be that cases without significant ulnar-sided soft tissue disruption are less likely to have this deformity preoperatively, and it may also be of less relevance in patients without significant ulnar-sided disruption. Further, there is evidence that in the $50-60 \%$ of patients who do not have a discrete DOB of the IOM, ${ }^{10,16}$ DRUJ instability may not result from this malreduction. Given that it is difficult at the time of surgery to quantify the degree of ulnar-sided soft tissue disruption or the presence of a discrete DOB of the IOM, it is even more important for surgeons to ensure that they correct the bony anatomy of the radius appropriately in every distal radius fracture fixation case.

\footnotetext{
Acknowledgments

We would like to acknowledge Dr Nick Daunt and Dominic Kennedy, Queensland X-Ray, for their assistance with the radiology dataset.
}

No benefits in any form have been received or will be received from a commercial party related directly or indirectly to the subject of this article.

\section{Conflict of Interest}

The Brisbane Hand and Upper Limb Research Institute received charitable donations from LMT, Medartis, and Depuy. Mark Ross is a consultant to LMT, Integra, and Surgicraft and is on speakers bureaus for Integra, Depuy, Trimed, Medartis, Synthes/AO. Livio Di Mascio, Fraser Taylor, Allen Cockfield, Susan Peters and Greg Couzens have no conflicts of interest to declare.

\section{Ethical Review Committee Statement}

This study received ethical review (1700QA) by the Mater Health Services Human Research Ethics Committee.

\section{Location}

All work was conducted at the Brisbane Hand and Upper Limb Research Institute, Brisbane Queensland, Australia.

\section{References}

1 Green DP. Pins and plaster treatment of comminuted fractures of the distal end of the radius. J Bone Joint Surg Am 1975;57(3): 304-310

2 Palmer AK. Fractures of the distal radius. In: Green DP, ed. Operative Hand Surgery, vol.2, 2nd ed. New York, NY: ChurchillLivingstone; 1988:991-1026

3 Altissimi M, Antenucci R, Fiacca C, Mancini GB. Long-term results of conservative treatment of fractures of the distal radius. Clin Orthop Relat Res 1986;206(206):202-210

4 Ward LD, Ambrose CG, Masson MV, Levaro F. The role of the distal radioulnar ligaments, interosseous membrane, and joint capsule in distal radioulnar joint stability. J Hand Surg Am 2000;25(2): 341-351

5 Gofton WT, Gordon KD, Dunning CE, Johnson JA, King GJW. Softtissue stabilizers of the distal radioulnar joint: an in vitro kinematic study. J Hand Surg Am 2004;29(3):423-431

6 Ross M, Heiss-Dunlop W. Volar angle stable plating for distal radius fractures. In: Slutsky DJ, ed. Principles and Practice of Wrist Surgery. Philadelphia, PA: WB Saunders; 2010:126-139

7 Lawler E, Adams BD. Reconstruction for DRUJ instability. Hand (NY) 2007;2(3):123-126

8 Hauck RM, Skahen J III, Palmer AK. Classification and treatment of ulnar styloid nonunion. J Hand Surg Am 1996;21(3):418-422

9 Sammer DM, Shah HM, Shauver MJ, Chung KC. The effect of ulnar styloid fractures on patient-rated outcomes after volar locking plating of distal radius fractures. J Hand Surg Am 2009;34(9): 1595-1602

10 Dy CJ, Jang E, Taylor SA, Meyers KN, Wolfe SW. The impact of coronal alignment on distal radioulnar joint stability following distal radius fracture. Proceedings of the Annual Meeting of the American Society for Surgery of the Hand (ASSH), October 2013; in review J Bone Joint Surg.

11 Fleiss J. The Design and Analysis of Clinical Experiments. New York, NY: John Wiley and Sons; 1986

12 Fujitani R, Omokawa S, Akahane M, Iida A, Ono H, Tanaka Y Predictors of distal radioulnar joint instability in distal radius fractures. J Hand Surg Am 2011;36(12):1919-1925 
13 Koh S, Andersen CR, Buford WL Jr, Patterson RM, Viegas SF. Anatomy of the distal brachioradialis and its potential relationship to distal radius fracture. J Hand Surg Am 2006;31(1):2-8

14 Rapley JH, Kearny JP, Schrayer A, Viegas SF. Ulnar translation, a commonly overlooked, unrecognized deformity of distal radius fractures: techniques to correct the malalignment. Tech Hand Up Extrem Surg 2008;12(3):166-169

15 Bronstein AJ, Trumble TE, Tencer AF. The effects of distal radius fracture malalignment on forearm rotation: a cadaveric study. J Hand Surg Am 1997;22(2):258-262
16 Noda K, Goto A, Murase T, Sugamoto K, Yoshikawa H, Moritomo H. Interosseous membrane of the forearm: an anatomical study of ligament attachment locations. J Hand Surg Am 2009;34(3): 415-422

17 Sagerman SD, Zogby RG, Palmer AK, Werner FW, Fortino MD. Relative articular inclination of the distal radioulnar joint: a radiographic study. J Hand Surg Am 1995;20(4):597-601

18 Metz VM, Gilula LA. Imaging techniques for distal radius fractures and related injuries. Orthop Clin North Am 1993;24(2): $217-228$

This article has been changed according to the Erratum published on February 26, 2014 (DOI: 10.1055/s-0034-1371547). The "Background" section of the abstract has been revised. 